\title{
effondrements spontanés et possibilité de mise en charge hydraulique
}

\author{
par \\ V. Maury \\ Société GEOSTOCK
}

L'étude de la stabilité de vieux travaux miniers et d'anciennes carrières nous ont conduit à réexaminer le phénomène d'effondrement spontané dont certaines exploitations sont le siège.

Lorsque l'extraction est faite par la méthode de chambres et piliers abandonnés à taux d'exploitation partielle, on observe parfois des ruptures et effondrements lents et progressifs; ceux-ci sont dus à une insuffisance de résistance, et la rupture, locale à l'origine, se bloque elle-même ou évolue progressivement, jusqu'à provoquer parfois la ruine d'un quartier.

Dans d'autres cas, en cours ou après la fin de l'exploitation, la mine ou la carrière peut être le siège d'un effondrement général, brutal, provoquant la ruine d'un quartier ou même d'une exploitation toute entière simultanément et se répercutant en surface.

Depuis 1971, on a donc repris l'analyse de nombreux effondrements dont les archives sont assez claires. La synthèse de leurs caractères communs (V. Maury 1979) et de leurs différences conduit à penser que dans certains cas, le toit peut se mettre en charge hydrauliquement, et cette mise en charge être responsable d'une rupture à évolution très rapide. Cette hypothèse permet d'expliquer de nombreuses constatations faites sur des effondrements de ce type.

\section{Principaux caractères des effondrements spontanés}

Ils ont été décrits et analysés dans un article de Tincelin et Sinou (1962), à propos d'effondrements spontanés survenus en Lorraine.

Dans les mines de fer de Lorraine, les exploitations sujettes à des effondrements spontanés présentent toutes les caractères communs suivants.

- Taux d'extraction de 60 à $75 \%$; exploitation par chambres et piliers; piliers de section voisine du carré ou du losange, de hauteur inférieure ou égale à $5 \mathrm{~m}$.
- Toit immédiat excellent et solide.

- Hauteur du recouvrement voisine de $140 \mathrm{~m}$.

- Zone exploitée par petits piliers limitée par des régions non exploitées, ou faiblement exploitées, qu'on appelle bords fermes; la distance des bords fermes est dans tous les cas égale ou légèrement supérieure à la hauteur du recouvrement. Ces "bords fermes" sont appelés "étau de masse" dans les carrières de la région parisienne.

(Ces principaux caractères sont présentés sur la fig. 1.) Les effondrements eux-mêmes présentent des traits communs :

- ils sont brutaux et inopinés sur toute la zone exploitée par les petits piliers générant une véritable secousse sismique et un souffle puissant et meurtrier; seuls les piliers de bordures sont préservés pendant quelques jours;

- ils provoquent une subsidence et des crevasses au jour jusqu'à 1 m d'ouverture, à l'aplomb des bords fermes;

— ils sont précédés d'une mise en charge de la zone centrale, n'ayant toutefois rien de spectaculaire ni alarmant.

D'autres coĩncidences ont été notées :

- présence de carrières de pierres à bâtir dans les environs;

- effondrements survenant après une longue période pluvieuse la plupart du temps.

Le mécanisme proposé par Tincelin et Sinou (1962) est alors le suivant:

Tant que la largeur d'exploitation reste faible, le toit constitué d'un ensemble de dalles agit comme une dalle épaisse, même si le taux d'extraction est relativement élevé; les piliers sont alors déchargés d'une partie du poids de recouvrement.

Lorsque la largeur d'exploitation augmente, et que les piliers continuent à se déformer, soit par fluage, soit 


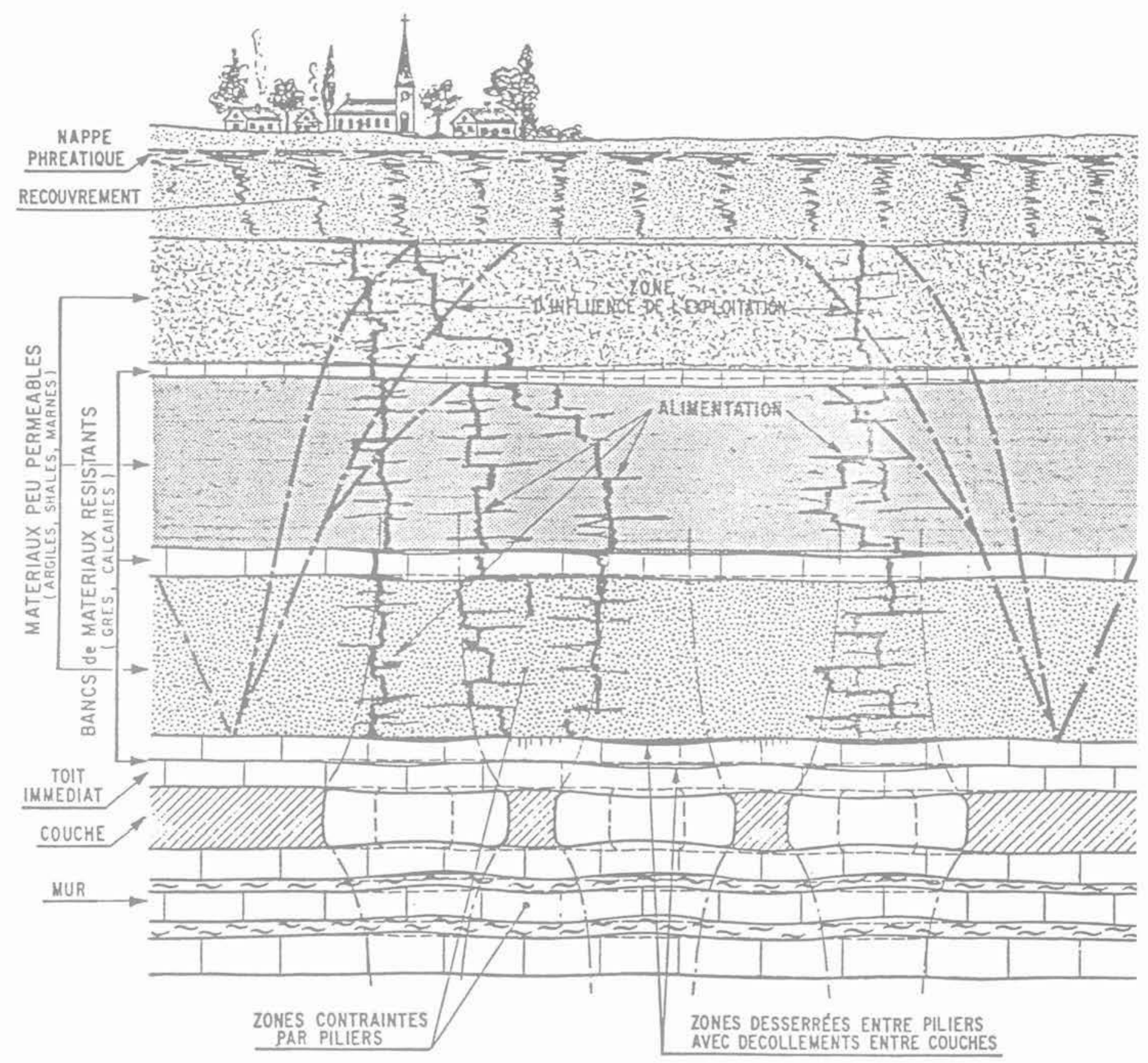

Fig. 1 Le champ de déplacement avec un taux d'extraction supérieur à $60 \%$ devient celui correspondant au poinçonne. ment d'un ensemble de piliers sur un milieu stratifié. I/ se produit un déplacernent d'ensemble au-dessus de la zone d'influence de I'exploitation.

12 piliers seuls ont été figurés pour faciliter la lecture du schéma. En place, la largeur d'exploitation est de l'ordre de la pro. fondeur, ce qui correspond à un nombre bien plus élevé de piliers).

pour une autre raison telle qu'augmentation du taux d'extraction, les bancs de recouvrement sont alors trop sollicités en flexion et se désolidarisent par glissement le long des joints de stratification, au droit des bords fermes. Les piliers sont alors surchargés et la rupture apparaît comme la libération de l'énergie emmagasinée par résistance au frottement le long des joints.

Cette interprétation permet d'expliquer de nombreuses constatations faites en Lorraine, et surtout d'éviter l'apparition de nouveaux effondrements.
De nombreuses études de stabilité nous ont incité à examiner d'autres ruptures, et des observations complémentaires peuvent y être faites.

\section{Observations complémentaires}

On a repris l'analyse détaillée de rapports d'effondrements de la mine de sel de Varangéville (1873), de la carrière de pierres à bâtir de Chancelade (1885), des charbonnages de Coal Brooks (Afrique du Sud 1960), 
de la carrière de calcaire à ciment de Champagnole (1964), d'effondrements survenus dans la région parisienne, dans la craie (Clamart 1961); enfin des données ont pu être rassemblées sur un effondrement survenu récemment dans une carrière de schistes argileux.

On peut en tirer les conclusions suivantes:

2.1 Tous les effondrements surviennent en milieu sédimentaire, à pendage faible ou nul.

2.2 Le comportement du matériau semble indifférent, comme en témoigne la liste ci-dessus, à laquelle il faudrait adjoindre vraisemblablement les phosphates, certains minerais de plomb, zinc, etc.

2.3 La nature du toit n'est pas non plus déterminante, mais on note sa rigidité et sa résistance au moins égale à celle de la couche exploitée et très souvent supérieure: alternance sel-marne à Varangéville, calcaire à Chancelade, alternance schisto-gréseuse à Coal Brooks, à schistes prédominants, calcaire de même nature que la couche à Champagnole, calcaire au toit de la carrière d'argilite, marnes et argiles à Clamart.

Aucune analogie ne peut être trouvée dans la lithologie du toit :

tous genres de matériaux à Varangéville, prédominance de grès à Coal Brooks, marno-calcaires à Champagnole, argilites et grès au-dessus de la carrière de schistes argileux, marnes et argiles à Clamart.

La hauteur de la couche est toujours supérieure à $5 \mathrm{~m}$.

2.4 Ces effondrements sont tous du même type, spontané, brutal, caractérisés par :

- souffle très violent, et génération d'une véritable secousse sismique artificielle;

- mise en charge de piliers préalable, notée à Varangéville, dans la carrière de schistes argileux et Champagnole et même à Chancelade (signes prémonitoires au cours de l'été 1885), mais peu à Coal Brooks d'après les documents publiés, et donc non systématique;

- dégâts de fonds et de surface, et effets très meurtriers du fait de l'absence de signes prémonitoires.

2.5 Les exploitations où surviennent ces effondrements ne sont pas connues pour avoir posé de problèmes de venues d'eau.

L'explication de ces effondrements par rupture en chaîne de piliers est incapable d'expliquer la brièveté du phénomène, et son occurence dans des contextes si variés. II semble, en outre, que ces effondrements surviennent parfois indépendamment de la rupture des piliers.

II faut toutefois signaler un écart entre ces observations et celles faites dans les mines de fer:

- La hauteur de recouvrement, bien de l'ordre de $140 \mathrm{~m}$ à Coal Brooks comme dans les schistes argileux, de 160 à $200 \mathrm{~m}$ à Varangéville, n'était de $152 \mathrm{~m}$ à Champagnole qu'au droit du Mont-Rivel, et l'effondrement s'est étendu jusqu'à des zones à très faible recouvrement. Le cas de Chancelade est encore plus net : la hauteur de recouvrement n'était que de $69 \mathrm{~m}$ au maximum, et là encore, l'effondre- ment s'est étendu jusqu'à des zones de 10 ou $25 \mathrm{~m}$ de recouvrement. A Clamart, l'effondrement s'est produit sous des zones de 40 à $70 \mathrm{~m}$ de recouvrement.

il semble donc que la hauteur de recouvrement de $150 \mathrm{~m}$ ne soit pas une condition nécessaire d'effondrement spontané. A part cette circonstance, les autres conditions sont cohérentes avec celles rencontrées dans les mines de fer.

L'explication du phénomène par libération brutale du frottement le long des bancs du toit au pourtour de l'exploitation nécessiterait, pour être applicable à tous les effondrements analysés, une lithologie comparable, du moins une disposition analogue. Or rien de tel ne peut y être trouvé. Le fait, signalé par Tincelin que les effondrements aient été précédés d'une période pluvieuse nous a incité à examiner le comportement possible du toit, d'un point de vue hydraulique.

\section{Mécanisme initiateur possible}

Les massifs sujets aux effondrements spontanés présentent du fait de leur stratification et de leur hétérogénéité, des alternances :

- de matériaux très peu perméables, parfois plastiques, de faible résistance au cisaillement, tels que marnes, argiles.

- et de matériaux plus résistants mécaniquement, parfois fracturés, formant une ossature plus rigide, tels que calcaires, grès, dolomies.

L'ensemble du massif est parcouru par la fissuration inhérente à la quasi-totalité des massif́s rocheux.

Que se passe-t-il alors lorsque l'exploitation minière progresse, au point de vue hydraulique, dans le toit?

Tant que les taux d'extraction restent faibles, et les piliers de faible hauteur, le champ de déplacement induit autour des galeries est celui de galeries isolées, c'est-à-dire qu'il n'y a pas de déplacements verticaux appréciables dans l'axe du pilier. En fait, il y a déjà dans le toit immédiat des galeries des décollements entre les dalles correspondant au poinçonnement du pilier dans le milieu stratifié. Ceux-ci forment un véritable réseau de vides interconnectés bien ou mal drainés. Ils peuvent être désaturés car non alimentés et protégés par les horizons très peu perméables du haut toit.

Lorsque le taux d'extraction augmente par reprise des piliers, (cas de Varangéville, Champagnole, Chancelade), ou que la hauteur des piliers augmente, (Champagnole, Coal Brooks, schistes argileux), les déplacements dans le pilier se rapprochent d'un champ monoaxial, et les composantes verticales peuvent atteindre plusieurs millimètres.

En fait, les composantes verticales du déplacement dans les piliers peuvent encore augmenter pour de nombreuses raisons :

- taux d'extraction fort et simple déplacement élastique;

- taux d'extraction fort et déplacement ou prérupture correspondant à une phase plastique ou fluage (Champagnole);

- augmentation de hauteur pour reprise de minerai au mur (schistes argileux);

- imprégnation d'eau de matériaux sensibles au mur (Varangéville); 
- imprégnation d'eau de la couche elle-même par inondation, et décroissance du module (certaines carrières du bassin parisien).

A ce moment, il se produit un déplacement d'ensemble du recouvrement (fig. 1); celui-ci s'accompagne de glissements des bancs les uns par rapport aux autres en particulier aux encastrements de la zone exploitée. (mécanisme Tincelin), l'ouverture de la microfissuration, ainsi que du jeu des diaclases. Les piliers poinçonnent le milieu stratifié dans une colonne pénétrant profondément dans le milieu. Le toit immédiat se déforme en restant dans le domaine élastique comme le montre un simple calcul de dalles (Maury 1970, 1979) et reste suffisamment continu et peu perméable pour ne permettre qu'un drainage insuffisant ou nul. La fibre comprimée à l'intérieur du toit peut contribuer à resserrer la fissuration et freiner le drainage.

II suffit alors d'une mise en communication avec un des niveaux aquifères ou perméables du recouvrement pour que :

- I'alimentation se fasse;

- la saturation s'obtienne, alors que le drainage par les travaux miniers reste impossible, ou insuffisant du fait de la bonne qualité du toit.

Dès que la saturation est obtenue, la mise en charge peut être très rapide et s'établir simultanément sur de très vastes étendues, la charge hydrostatique affectant tout le toit latéralement et en hauteur.

Les diverses dalles du toit immédiat chargées par leur. poids propre, éventuellement une hauteur de terrain desserrée peu cohérente, et les contraintes horizontales, sont alors brutalement et toutes soumises à la pression hydrostatique. Les composantes horizontales des contraintes géostatiques peuvent alors aider les dalles du toit à supporter l'effet d'une partie de cette charge diminuant les tractions induites par les flexions mais pas les efforts tranchants.

Dès que la charge hydraulique complète s'exerce, les cisaillements aux encastrements et éventuellement les tractions induites deviennent trop forts pour être compensés par un système de contraintes horizontales normal c'est-à-dire inférieures ou égales au poids des terres.

Le régime de pression interstitielle induit par-la charge hydraulique non drainée a un deuxième effet, désastreux également : les joints de stratification du toit voient leur résistance au cisaillement s'effondrer du fait de cette pression interstitielle. Cet effet est certainement beaucoup plus nocif que celui de lubrification des joints, dont le matériau était vraisemblablement saturé ou proche de la saturation, et joue dans le sens d'une chute très rapide de la résistance au cisaillement aux encastrements des dalles.

La rupture du toit, immédiat le plus souvent, survient et la propagation peut se faire de deux façons, éventuellement simultanées :

- remontée de l'effondrement dans le toit, les dalles du toit profond étant brutalement soumises aux pressions interstitielles que la première rupture n'a pas soulagées;

- extension latérale de la rupture soit par génération d'une surpression interstitielle dynamique et rupture des autres dalles, soit rupture des autres dalles par interruption brutale de la composante géostatique horizontale;

Une troisième possibilité existe encore: comporte- ment de la partie centrale du toit comme une dalle épaisse monobloc, insuffisamment soutenue, et passant d'une position d'équilibre stable à instable.

Pour trancher entre ces divers mécanismes, it conviendrait de disposer d'observations détaillées des ruptures, qui sont rares et dangereuses. Celles en notre possession font actuellement pencher pour une combinaison des deux premiers modes de propagation.

Les effondrements analysés ont été examinés sous l'angle de ce mécanisme initiateur possible par mise en charge hydraulique.

II est actuellement possible que la rupture initiale au-dessus de certaines cavités lessivées soit du même type, une déformation excessive du toit immédiat due à une pression insuffisante de la saumure mettant en charge hydraulique un horizon intermédiaire.

\section{Examen des effondrements sous l'angle du mécanisme initiateur proposé}

\subsection{L'influence du réseau hydrogéologique}

Selon le mécanisme évoqué, l'effondrement peut être provoqué par les charges de nappes phréatiques elles-mêmes, ou par une surcharge de celles-ci : si l'excavation draine suffisamment le toit, soit accidentellement par la fissuration, soit par la matrice, celui-ci peut supporter une partie de la charge hydraulique des nappes phréatiques, mais il peut très bien ne plus supporter une surcharge de celles-ci, due à la pluviométrie : dans les mines de fer de Lorraine, la liaison est très probable avec la pluviométrie (Tincelin 1962) avec un décalage - le temps de la mise en charge; le phénomène est possible également dans la carrière de schistes argileux (très importantes précipitations 3 semaines avant l'éffondrement), bien qu'il semble qu'il n'y ait eu aucun drainage permanent par l'excavation.

II s'explique ainsi que la liaison avec la pluviométrie soit aléatoire. Les informations disponibles à Varangéville et Champagnole confirment ceci.

A Clamart, une alimentation artificielle (par fuite de canalisation de surface) a dû également jouer, en plus de très fortes précipitations ayant précédé la rupture.

Notons que l'apparition de forts débits peut avoir lieu ou non, pressions et débits étant complètement indépendants, le débit ne dépendant que de la puissance et de l'alimentation de l'aquifère de surface. La mise en charge peut donc se faire aussi à partir d'aquifères peu ou mal alimentés, peu susceptibles de créer des conditions de venues d'eau soudaines auxquelles les mineurs sont habitués dans certaines exploitations. C'est sans doute la raison pour laquelle ce mécanisme n'a jamais été évoqué pour les effondrements spontanés.

\subsection{L'extension des effondrements à des zones à faible recouvrement}

La perméabilité horizontale étant augmentée par le jeu de dalles du toit du fait du poinçonnement des piliers dans le milieu stratifié où se trouvent des horizons déformables, la mise en charge hydraulique des dalles peut se prolonger très loin horizontalement, y compris dans des zones où le recouvrement devient faible : des pressions de l'ordre de 0,5 MPa peuvent s'exercer ainsi jusqu'à des zones où le recouvrement n'est plus que de $25 \mathrm{~m}$. C'est ce qui s'est passé à Chancelade, et à 
Champagnole, et permet d'expliquer l'extension de la rupture à des zones à si faible recouvrement, inexplicable autrement.

La confirmation de perméabilité évoquée ci-dessus : perméabilité horizontale très grande par rapport à la perméabilité verticale, elle-même beaucoup plus forte que la perméabilité matricielle a été remarquablement confirmée dans le recouvrement d'une carrière souterraine de calcaires aux États-Unis, étudiée dans un tout autre but.

\subsection{La préservation des zones de bordures}

Dans ces zones, le taux d'extraction est plus faible, ou la hauteur des piliers est plus faible. Les galeries se déforment en régime de galeries isolées; les interstices entre les dalles du toit ne sont pas en communication hydraulique et le mécanisme initiateur ne se met pas en place : ceci est particulièrement net à Champagnole sur les bords de l'exploitation, mais surtout sur la limite Sud, où la partie d'exploitation assez ancienne (antérieure à 1960) tracée en chambres parallèles a été la seule à ne pas subir l'effondrement.

\subsection{L'absence d'effondrement dans certaines mines}

Il suffit qu'un élément nécessaire à la mise en charge du toit soit absent pour que l'exploitation ne soit pas soumise à ce type d'effondrement :

- qu'un drainage par le toit soit suffisant;

- qu'il se produise une décharge des aquifères de surfaces à flanc de colline;

- que la première rupture, localisée, suffise à décharger définitivement le toit hydrauliquement;

- qu'enfin un boulonnage, ou une «mauvaisen exploitation entame le toit, et suffise à empêcher la mise en charge possible.

C'est heureusement le cas de nombreuses exploitations menées en chambres et piliers.

A l'échelle des travaux miniers, les remèdes contre ces effondrements consistent essentiellement à piézométrer le toit, le drainer, et d'une façon plus générale à tout faire pour éviter l'apparition de pressions interstitielles.

\section{Conclusion}

La variété de matériaux et de structure lithologique dans lesquels les effondrements spontanés se manifestent fait émettre l'hypothèse d'un mécanisme initiateur dû à l'établissement d'une pression interstitielle dans le toit des exploitations: sous l'effet d'un taux d'extraction important, de piliers hauts ou du fluage du matériau, il se produit un déplacement d'ensemble du recouvrement, maintenant le toit immédiat résistant continu, empêchant ou freinant son drainage par les travaux miniers. Lorsque l'extension des travaux miniers devient suffisante, les déplacements induits dans le toit profond provoquent le jeu et l'ouverture de la stratification et de la fissuration. L'alimentation des joints ouverts dans le toit immédiat par le poinçonnement des piliers devient alors possible. Les nappes phréatiques de surface, statiques ou surchargées par la pluviométrie peuvent alors mettre en charge tout le toit jusqu'à ce qu'en un point les dalles du toit immédiat cassent, entraînant la rupture du toit profond. Le système de contraintes horizontales qui maintenait le toit immédiat en place est brutalement relâché et la rupture du toit peut se propager sur de très grandes étendues. Un mécanisme analogue peut faire claquer une dalle résistante du toit profond et aboutir aux mêmes effets.

Devant les risques que présentent ce mode d'effondrement, il serait souhaitable que les exploitants miniers au courant de telles manifestations, ou les jugeant possibles sur leur exploitation, fassent part de toutes les observations permettant de confirmer cette hypothèse ou d'en préciser les limites.

A l'échelle des travaux miniers, une prévention contre de telles ruptures meurtrières et ruineuses peut être obtenue moyennant une auscultation de si faible cout que son installation gagnerait à être examinée systématiquement.

\section{Références bibliographiques}

\section{Bibliographie générale}

Tincelin et Sinou «Effondrements brutaux et généralisés. Coups de toit». R.I.M. Avril 1962.

Maury V. "Mécanique des milieux stratifiés". Éd. Dunod 1970.

Maury V. "Effondrements spontanés". Synthèse d'observations et possibilité de mécanisme initiateur par mise en charge hydraulique. Revue de I'Industrie Minérale. Octobre 1979.

\section{Bibliographie sommaire relative aux effrondements}

CHANCELADE - 25 Octobre 1885

"La catastrophe de Chancelade, près de Périgueux ". "La Nature $*, 1886,1^{\text {er }}$ semestre.

COAL BROOKS - 21 Janvier 1960

"Quelques problèmes sur le contrôle des terrains et sur le soutènement de travaux par chambres et piliers". Revue Universelle des Mines $9^{e}$ série T, XX $n^{\circ} 11$ Novembre 1964, p. 309 à 322. 
